This is the author's final, peer-reviewed manuscript as accepted for publication. The publisher-formatted version may be available through the publisher's web site or your institution's library.

\title{
On cellular indecomposable property of semi-Fredholm operators
}

Guozheng Cheng, Xiang Fang

\section{How to cite this manuscript}

If you make reference to this version of the manuscript, use the following information:

Cheng, G., \& Fang, X. (2012). On cellular indecomposable property of semi-Fredholm operators. Retrieved from http://krex.ksu.edu

\section{Published Version Information}

Citation: Cheng, G., \& Fang, X. (2012). On the cellular indecomposable property of semi-Fredholm operators. Chinese Annals of Mathematics, Series B, 33(6), 903-908.

Copyright: ( $\odot$ The Editorial Office of CAM and Springer-Verlag 2012

Digital Object Identifier (DOI): doi:10.1007/s11401-012-0744-x

Publisher's Link: http://link.springer.com/article/10.1007/s11401-012-0744-x?null

This item was retrieved from the K-State Research Exchange (K-REx), the institutional repository of Kansas State University. K-REx is available at http://krex.ksu.edu 


\title{
On Cellular Indecomposable Property of Semi-Fredholm Operators
}

\author{
Guozheng Cheng \\ School of Mathematics \\ Wenzhou University \\ Wenzhou, Zhejiang, 325035, China \\ Email: chgzh09@gmail.com
}

\author{
Xiang Fang* \\ Department of Mathematics \\ Kansas State University \\ Manhattan, KS, 66502 \\ Email:xfang@math.ksu.edu
}

\begin{abstract}
In this paper we prove that an operator with the cellular indecomposable property has no singular points in the semi-Fredholm domain. Besides its own interests, this fills a gap in [3]. Our proof relies on the $4 \times 4$ matrix model of semi-Fredholm operators [2].
\end{abstract}

Keywords: cellular indecomposable property; semi-Fredholm operators; singular points.

In a series of three papers [3], [4], [5], R. Olin and J. Thomson introduced and studied the cellular indecomposable property (CIP) which has become a basic notion in operator theory. An operator $T \in B(H)$ has (CIP) if any two nontrivial invariant subspaces $M_{1}, M_{2} \subset H$ of $T$ have a nontrivial intersection $M_{1} \cap M_{2} \neq\{0\}$. Note that if $T$ has (CIP), then so does $T-\lambda$ for any $\lambda \in \mathbb{C}$ since $T$ and $T-\lambda$ have the same invariant subspace lattice.

The principle question underlying Olin and Thomson's research is what the spectral picture [6] of a CIP operator can look like. For instance, one can show that the Fredholm index of a CIP operator cannot be positive, hence the adjoint

*Partially supported by National Science Foundation Grant DMS 0801174 and Laboratory of Mathematics for Nonlinear Science, Fudan University. 
is quasi-triangular [1], [6]. It is easy to achieve index 0 or -1 , and it is still not known whether the index can be -2 or smaller.

Motivated by the spectral picture problem, Olin and Thomson made a thorough analysis of subnormal operators with (CIP). For general operators, they proved a result on semi-Fredholm operators (Lemma 4 in [3], see Theorem 1 in this paper) which is needed in the proof of the main result in [3]. Their proof of Lemma 4, however, contains a gap in handling singular points in the semi-Fredholm domain as explained below.

On the other hand, their result is almost certainly useful for further study of the spectral theory of a general CIP operator. This prompts us to find a complete proof and in this paper we prove a result (Theorem 2) which is enough to fill the gap and is of independent interests-we show that a CIP operator has no singularity at all.

Our main technical tool is the $4 \times 4$ matrix model of semi-Fredholm operators developed in [2].

Recall that a singular point $\lambda_{0} \in \rho_{F}(T)$ in the Fredholm domain $\rho_{F}(T)$ of an operator $T \in B(H)$ acting on a Hilbert space $H$ is a point $\lambda_{0}$ such that the dimension function of the kernel

$$
\lambda \rightarrow \operatorname{dim}(k e r(T-\lambda))
$$

is not continuous at $\lambda_{0}$. When $\lambda_{0} \in \rho_{s F}(T)$, the semi-Fredholm domain, $\lambda_{0}$ is singular if the projection $P_{k e r(T-\lambda)}$ does not converge to $P_{k e r\left(T-\lambda_{0}\right)}$ as $\lambda \rightarrow \lambda_{0}$ in the strong operator topology. In this paper, we mainly consider those singular points in the semi-Fredholm domain.

To overcome the complexity caused by a singular point, [3] used a translation argument: For a semi-Fredholm $T$, possibly singular at 0 , they replaced $T$ by $T-\lambda$ for some small $\lambda$ so they assume that $T$ is regular at 0 . However, they implicitly used the following argument: If $T$ is analytic, then so is $T-\lambda$. Here an operator $T$ is analytic if

$$
\cap_{k \geq 0} T^{k} H=\{0\} .
$$

See the first line and the last line of page 402 of [3]. This is not true as illustrated by the following one dimensional extension of a pure isometry $S \in B(H)$,

$$
T=\left(\begin{array}{ll}
0 & 0 \\
0 & S
\end{array}\right) \in B(\mathbb{C} \oplus H) .
$$


The statement of the following Theorem 1 is the same as Lemma 4 in [3].

Theorem 1. If $T$ is a semi-Fredholm operator such that

(1) the Fredholm index satisfies index $(T) \notin\{0,-1\}$, and

(2) $T$ is analytic, $\cap_{k \geq 0} T^{k} H=\{0\}$,

then $T$ is cellular decomposable, that is, it has no (CIP).

A close examination of the proof in [3] shows that the arguments there do not work for the above $T$ in (1). The obstacle is at the end of page 402: After a translation $T-\lambda$, the second analytic condition (2) in Theorem 1 is no longer satisfied. Moreover, [3] actually proved Theorem 1 under an extra condition

(3) $T$ has no singularity at 0.

The main result of this paper is the following.

Theorem 2. If the Hilbert space $H$ is infinite dimensional, $\operatorname{dim}(H)=\infty$, and $T \in B(H)$ is cellular indecomposable, then $T$ has no singular points in its semiFredholm domain.

So Theorem 1 follows from Theorem 2 and the proof of Olin-Thomson in [3]. Note that Theorem 2 does not hold on a finite dimensional Hilbert space, as illustrated by a single nilpotent Jordan block, which indeed has (CIP) and is singular at the origin.

Corollary 3. If $T \in B(H)$ is an operator with the cellular indecomposable property, and $T$ is semi-Fredholm, then $T$ has the following matrix decomposition,

$$
T=\left(\begin{array}{cc}
T_{1} & A \\
0 & T_{2}
\end{array}\right)
$$

Here the decomposition is with respect to $H_{1} \oplus H_{1}^{\perp}$, with $H_{1}=\cap_{k \geq 1} T^{k} H$, $T_{1} \in B\left(H_{1}\right)$ is invertible, and $T_{2}$ is a pure shift.

Recall that a pure shift is a left-invertible operator which is also analytic [2]. The proof of Corollary 3 is essentially contained in the proof of Theorem 2.

It is an interesting question to see when the $T_{1}$ entry in (2) is indeed void. If index $(T) \leq-2$, then Theorem 1 implies that $T_{1}$ cannot be void. Again, we do not know whether index $(T) \leq-2$ can happen for a CIP operator. 
The rest of this paper is devoted to the proof of Theorem 2 .

Proof of Theorem 2. We first recall the $4 \times 4$ upper-triangular matrix model of semi-Fredholm operators developed in [2] which we rely on heavily.

For any semi-Fredholm $T \in B(H)$ we can decompose $H=H_{1} \oplus H_{2} \oplus H_{3} \oplus H_{4}$ into the direct sum of four closed subspaces, with some components possibly void, such that the associated matrix of $T$ has the form

$$
T=\left(\begin{array}{cccc}
T_{1} & * & * & * \\
0 & T_{2} & * & * \\
0 & 0 & T_{3} & * \\
0 & 0 & 0 & T_{4}
\end{array}\right)
$$

The properties of $T_{1}, T_{2}, T_{3}, T_{4}$ which we will need are listed below.

(i) $T_{4}$ is a pure shift semi-Fredholm operator. See the definition after Corollary 3. Or, to be more speficic, recall that a semi-Fredholm operator $S \in B(K)$ is a pure shift if

(a) $\operatorname{ker}(S)=\{0\}$, and

(b) $S$ is analytic, $\cap_{k \geq 0} S^{k} K=\{0\}$.

In particular, if $S$ is a pure shift, then $\operatorname{ker}\left(S^{*}\right) \neq\{0\}$ and $\operatorname{dim}\left(k e r\left(S^{*}-\lambda\right)\right)$ is a constant on a small open neighborhood of the origin by general Fredholm theory.

(ii) $T_{1}^{*}$ is a pure shift.

(iii) $T_{2}$ is invertible,

(iv) $T_{3}$ is a finite nilpotent matrix. In particular,

$$
\operatorname{dim}\left(H_{3}\right)=N<\infty
$$

It follows that

$$
T_{3}^{N}=0 .
$$

These two conditions will play important roles in the proof.

(v) The origin 0 is a singular point in the semi-Fredholm domain of $T$ if and only if $H_{3} \neq\{0\}$. So our goal is to show $H_{3}=\{0\}$. 
First we show that $H_{1}=\{0\}$. Otherwise, $H^{\prime}=k e r\left(T_{1}\right) \neq\{0\}$ is a nontrivial invariant subspace of $T$. Since $T_{1}^{*}$ is a pure shift,

$$
\operatorname{dim}\left(\operatorname{ker}\left(T_{1}\right)\right)=\operatorname{dim}\left(\operatorname{ker}\left(T_{1}-\lambda\right)\right)
$$

when $\lambda$ is small enough, but nonzero, we have

$$
H^{\prime \prime}=\operatorname{ker}\left(T_{1}-\lambda\right) \neq\{0\}
$$

to be another nontrivial invariant subspace of of $T_{1}$, hence of $T$. Clearly $H^{\prime} \cap H^{\prime \prime}=\{0\}$ since they consist of eigenvectors of different eigenvalues. This is a contradiction since $T$ has (CIP).

Next we show that at most one of $H_{2}$ and $H_{3}$ can be nonzero. Otherwise, $H_{2}$ is a nontrivial invariant subspace. Since $H_{3}$ is nonzero, by (v) above, 0 is a singular point of $T$, hence

$$
\operatorname{ker}(T) \neq\{0\}
$$

which is another nontrivial invariant subspace. Since $T_{2}=\left.T\right|_{H_{2}}$ is invertible, $T$ is bounded below on $H_{2}$. It follows that $H_{2} \cap \operatorname{ker}(T)=\{0\}$. Again a contradiction with (CIP).

If $H_{3}=\{0\}$, then we are done.

Next we assume that $H_{2}=\{0\}$, and $H_{3}$ is a nontrivial invariant subspace. In this case, $\mathrm{H}=\mathrm{H}_{3} \oplus H_{4}$.

Since $\operatorname{dim}(H)=\infty$ and $\operatorname{dim}\left(H_{3}\right)=N<\infty$, we know that $H_{4}$ is nontrivial. Since $T_{4}$ is a pure shift, we can choose a unit vector

$$
k \in \operatorname{ker}\left(T_{4}^{*}\right)
$$

and let $H_{k} \subset H$ denote the invariant subspace generated by $\left(\begin{array}{l}0 \\ k\end{array}\right)$ under the action of $T$.

Claim: $H_{k} \cap H_{3}=\{0\}$.

This will be in contradiction with (CIP), so it follows $H_{3}=\{0\}$, and we are done then. The rest of the proof is devoted to prove this claim. 
Next we assume that there is a sequence of polynomials $p_{t}(z) \in \mathbb{C}[z]$ such that

$$
\lim _{t \rightarrow \infty} p_{t}(T)\left(\begin{array}{l}
0 \\
k
\end{array}\right)=\left(\begin{array}{l}
e \\
0
\end{array}\right) \in H_{k} \cap H_{3},
$$

and we wish to show $e=0$.

Write

$$
T=\left(\begin{array}{cc}
T_{3} & A \\
0 & T_{4}
\end{array}\right)
$$

for some $A \in B\left(H_{4}, H_{3}\right)$ and for any polynomial

$$
p(z)=a_{0}+a_{1} z+\cdots+a_{n} z^{n},
$$

we write

$$
p(T)\left(\begin{array}{l}
0 \\
k
\end{array}\right)=\left(\begin{array}{cc}
p\left(T_{3}\right) & B_{p} \\
0 & p\left(T_{4}\right)
\end{array}\right)\left(\begin{array}{l}
0 \\
k
\end{array}\right)=\left(\begin{array}{c}
B_{p} k \\
p\left(T_{4}\right) k
\end{array}\right) .
$$

Here $B_{p}$ is a noncommutative polynomial of $T_{3}, A$ and $T_{4}$. If we can show that for any polynomial $p$,

$$
\left\|B_{p} k\right\| \leq C\left\|p\left(T_{4}\right) k\right\|
$$

for some constant $C$, independent of $p$, then we can conclude that $e=0$.

Without loss of generality, we assume

$$
n \geq N=\operatorname{dim}\left(H_{3}\right)
$$

since otherwise we can choose

$$
a_{n+1}=\cdots=a_{N}=0,
$$

so that $p$ is formally of degree $N$. This will make the bookkeeping in the proof of (8) easier. Equation (8) is a key step toward the proof of (6).

Next we calculate $B_{p}$ by direct calculation. For any $i=1,2, \cdots, N$, let

$$
B_{i}=a_{i} T_{3}^{i-1} A+a_{i+1} T_{3}^{i-1} A T_{4}+\cdots+a_{n} T_{3}^{i-1} A T_{4}^{n-i} .
$$

By using

$$
T_{3}^{N}=0
$$


and by writing out all terms in $B_{p}$, we have

$$
B_{p}=B_{1}+\cdots+B_{N}
$$

The proof of (8) involves some work on bookkeeping, but there is nothing challenging. In writing out all terms of $B_{p}$, one just needs to keep (7) in mind.

Note that $N=\operatorname{dim}\left(H_{3}\right)$ is independent of $p=p(z)$. So it suffices to show that for each $i=1,2, \cdots, N$,

$$
\left\|B_{i} k\right\| \leq C\left\|p\left(T_{4}\right) k\right\|
$$

for some constant $C$, independent of $p$. Let

$$
B_{i}^{\prime}=a_{i}+a_{i+1} T_{4}+\cdots+a_{n} T_{4}^{n-i}
$$

then

$$
B_{i}=T_{3}^{i-1} A B_{i}^{\prime}
$$

hence it suffices to show

$$
\left\|B_{i}^{\prime} k\right\| \leq C\left\|p\left(T_{4}\right) k\right\|
$$

for some constant $C$, independent of $p$.

Next we show (9) by induction. First for $i=1$. Since $T_{4}$ is a pure shift, it is bounded below, so we assume

$$
\left\|T_{4} x\right\| \geq c\|x\|
$$

for some $c>0$ and any $x \in H_{4}$.

Write

$$
p\left(T_{4}\right) k=a_{0} k+T_{4}\left(a_{1}+a_{2} T_{4}+\cdots+a_{n} T_{4}^{n-1}\right) k .
$$

By our choice of $k, k \perp T_{4} H_{4}$, so we have

$$
\begin{aligned}
\left\|p\left(T_{4}\right) k\right\|^{2} & =\left\|a_{0} k\right\|^{2}+\left\|T_{4}\left(a_{1}+a_{2} T_{4}+\cdots+a_{n} T_{4}^{n-1}\right) k\right\|^{2} \\
& \geq c^{2}\left\|\left(a_{1}+a_{2} T_{4}+\cdots+a_{n} T_{4}^{n-1}\right) k\right\|^{2},
\end{aligned}
$$

which is the case of $i=1$ for (9).

Now replace $p(z)$ by $q(z)=a_{1}+a_{2} z+\cdots+a_{n} z^{n-1}$, and apply the $i=1$ case of (9) to $q(z)$, one obtains the $i=2$ case of $(9)$ for $p(z)$, with a different constant $C$. Keep iterating this process and the proof of (9), hence the whole proof, can be completed. 


\section{References}

[1] C. Apostol, C. Foias, and D. Voiculescu, Some results on non-quasitriangular operators. IV, Rev Roumaine Math. Pures Appl. 18 (1973), 487-514.

[2] X. Fang, Samuel multiplicity and the structure of semi-Fredholm operators, Adv. in Math. 186 (2004), 411-437.

[3] R. Olin, J. Thomson, Cellular-indecomposable subnormal operators, Integral Equation and Operator Theory, 7 (1984), 392-430.

[4] R. Olin, J. Thomson, Cellular-indecomposable subnormal operators II, Integral Equation and Operator Theory, 9 (1986), 600-609.

[5] R. Olin, J. Thomson, Cellular-indecomposable subnormal operators III, Integral Equation and Operator Theory, 29 (1997), 116-121.

[6] C. Pearcy, Some Recent Developments in Operator Theory, CBMS series, 36, Amer. Math. Soc. Providence, 1975. 